\title{
Images in Practice: Successful Recapture of Spinal Cord Stimulator Paresthesia Coverage via Generator Reprogramming in a Patient with a Severely Displaced Percutaneous Trial Lead
}

Ruben H. Schwartz • Ivan Urits • Marc Solomon • Vwaire Orhurhu •

Alan D. Kaye · Omar Viswanath (iD

Received: November 22, 2019 / Published online: December 27, 2019

(C) The Author(s) 2019

Keywords: Device programming; Fluoroscopy; Recapture; Spinal cord stimulation

\section{Key Summary Points}

Migration of percutaneously implanted leads during spinal cord stimulator (SCS) trial is a significant complication that can substantially decrease the efficacy of spinal stimulation and subsequently affect the overall efficacy of the trial period.

The ability to recognize this via fluoroscopy and troubleshoot accordingly can potentially salvage the trial period still resulting in a successful overall trial.

Enhanced Digital Features To view enhanced digital features for this article go to https://doi.org/10.6084/ m9.figshare.11336627.

R. H. Schwartz

Department of Anesthesiology, Mount Sinai

Medical Center, Miami, FL, USA

I. Urits · V. Orhurhu

Department of Anesthesia, Critical Care, Pain

Medicine Harvard Medical School, Beth Israel

Deaconess Medical Center, Boston, MA, USA

M. Solomon · O. Viswanath $(\bowtie)$

Valley Anesthesiology and Pain Consultants,

Envision Physician Services, Phoenix, AZ, USA

e-mail: viswanoy@gmail.com
Interventional pain medicine physicians should be quick to utilize fluoroscopy to assess location of the percutaneous leads during the trial period whenever there is a sudden, acute loss of paresthesia coverage.

\section{IMAGES IN PRACTICE}

Migration of percutaneously implanted leads during spinal cord stimulator (SCS) trial is a significant complication that can substantially decrease the efficacy of spinal stimulation and subsequently affect the overall efficacy of the trial period. The ability to recognize this via fluoroscopy and troubleshoot accordingly can potentially salvage the trial period, and still result in a successful overall trial. The overall
A. D. Kaye
Department of Anesthesiology, Louisiana State University Health Sciences Center, New Orleans, LA, USA
O. Viswanath
Department of Anesthesiology, University of Arizona College of Medicine, Phoenix, Phoenix, AZ, USA
O. Viswanath
Department of Anesthesiology, Creighton
University School of Medicine, Omaha, NE, USA 
incidence of lead migration has been reported to be $13-22 \%$ [1]. Many factors have been associated with increased risk of migration. These include premature activity after placement, female sex, hardware manipulation, and lead anchoring technique [2].

Figure $1 \mathrm{a}, \mathrm{b}$ demonstrates the dramatic migration of percutaneous SCS leads on live fluoroscopic imaging during an initial trialing period and successful recapture of paresthesia coverage via reprogramming of the SCS generator. The patient was a 69 -year-old male who underwent uncomplicated percutaneous lead implantation for an SCS trial (Fig. 1a), and subsequently experienced an acute loss of paresthesia coverage during physical activity. The patient provided their informed consent to publish the article and all procedures were conducted as part of standard care/treatment. Figure $1 \mathrm{~b}$ demonstrates a profound caudal migration of the right lead from a T8 level initially to a T12 vertebral level. In this patient, revision of his percutaneous leads was avoided through the successful recapture of paresthesia coverage, using alternative SCS programming. Following reprogramming, the patient noted a

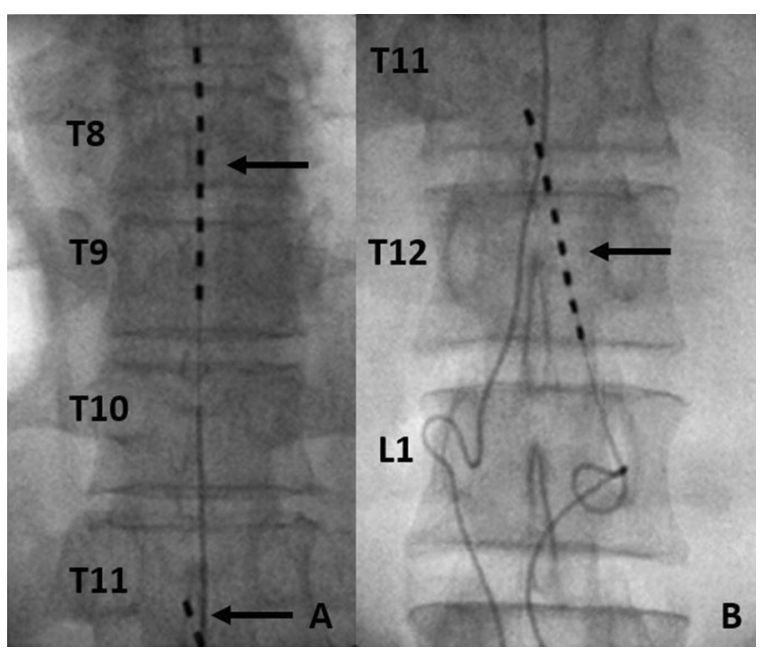

Fig. 1 a Intra-operative anteroposterior fluoroscopic imaging taken during permanent spinal cord stimulator (SCS) generator implantation. These radiographic images demonstrate a maintained position of the right percutaneous lead spanning vertebral levels T8 and T9 and a caudally displaced left percutaneous lead at the vertebral levels T11 and T12. b Final displaced position of the left SCS lead complete return of paresthesia coverage and resultant pain relief. Though lead migration is a relatively common complication of SCS therapy, minor, or as in this case, profound changes in lead position may not necessitate lead revision but rather may be managed through reprogramming. Interventional pain medicine physicians should be quick to utilize fluoroscopy to assess location of the percutaneous leads during the trial period whenever there is a sudden, acute loss of paresthesia coverage.

\section{ACKNOWLEDGEMENTS}

Funding. No funding or sponsorship was received for this study or publication of this article.

Authorship. All named authors meet the International Committee of Medical Journal Editors (ICMJE) criteria for authorship for this article, take responsibility for the integrity of the work as a whole, and have given their approval for this version to be published.

Disclosures. Ruben H. Schwartz, Ivan Urits, Marc Solomon, and Vwaire Orhurhu have nothing to disclose. Alan D. Kaye is a member of the journal's Editorial Board. Omar Viswanath is the Section Editor for Images in Practice.

Compliance with Ethics Guidelines. The patient provided their informed consent to publish the article and all procedures were conducted as part of standard care/treatment.

Open Access. This article is distributed under the terms of the Creative Commons Attribution-NonCommercial 4.0 International License (http://creativecommons.org/licenses/ by-nc/4.0/), which permits any noncommercial use, distribution, and reproduction in any medium, provided you give appropriate credit to the original author(s) and the source, provide a link to the Creative Commons license, and indicate if changes were made. 


\section{REFERENCES}

1. Gazelka HM, Freeman ED, Hooten WM, et al. Incidence of clinically significant percutaneous spinal cord stimulator lead migration. Neuromodulation. 2015;18(2):123-5.
2. Osborne Michael D, Ghazi Salim M, Palmer Scott C, Boone Katherine M, Sletten Christopher D, Nottmeier Eric W. Spinal cord stimulator-trial lead migration study. Pain Med. 2011;12(2):204-8. 